\title{
¿Hay realmente inteligencia animal? Una revisión filosófica.
}

\section{Is there really animal intelligence? A philosophical review.}

\author{
DOI: $10.32870 /$ sincronia.axxv.n80.11b21
}

\begin{abstract}
Víctor Hugo Gutiérrez Luna
Maestría en Pensamiento Crítico y Hermenéutica. Universidad Autónoma de Zacatecas (MÉXICO)

CE: victorluna@uaz.edu.mx

Juan Reyes Juárez

Doctorado en Filosofía e Historia de las Ideas. Universidad Autónoma de Zacatecas (MÉXICO)

CE: juanrokyireyes@gmail.com
\end{abstract}

\section{Esta obra está bajo una Licencia Creative Commons Atribución-NoComercial 4.0 Internacional}

Recibido: 31/03/2021

Revisado: 04/05/2021

Aprobado: 18/06/2021

\section{RESUMEN}

En el contexto de la investigación filosófica acerca de la inteligencia animal hay distintas tradiciones que niegan que los animales no humanos sean inteligentes. En este artículo mencionamos algunas de estas tradiciones, tales como el mecanicismo cartesiano y el conductismo. Sin embargo, centraremos nuestra atención en las propuestas de los filósofos analíticos John McDowell y Donald Davidson como representativas de esta tradición filosófica. Su idea principal es que al no poseer un lenguaje como el de los seres humanos, el resto de los animales no pueden ser racionales $y$, por ende, tampoco inteligentes.

Nuestra posición es que dicha tradición analítica ignora rotundamente la evidencia científica y filosófica en su contra. Daremos algunos datos relevantes a favor de la inteligencia animal. Además, daremos cuenta de una tendencia que se manifiesta cada vez con mayor fuerza entre los etólogos según la cual hay una continuidad entre la inteligencia animal y la humana, considerando a esta última como el resultado de un proceso evolutivo $y$, por tanto, como resultado de una serie de habilidades adquiridas por distintas especies en algún momento de su conformación. 
Palabras claves: Inteligencia animal. Racionalidad. Animales.

\section{ABSTRACT}

In the context of philosophical research on animal intelligence, there are different traditions that deny that nonhuman animals are intelligent. In this article we mention some of these traditions, such as Cartesian mechanism and behaviorism. However, we will focus our attention on the proposals of the analytical philosophers John McDowell and Donald Davidson as representative of this philosophical tradition. His main idea is that by not having a language like that of human beings, the rest of the animals cannot be rational and, therefore, not intelligent either.

Our position is that such an analytical tradition flatly ignores the scientific and philosophical evidence against it. We will give some relevant data in favor of animal intelligence. In addition, we will give an account of a trend that is manifested with increasing force among ethologists according to which there is a continuity between animal and human intelligence, considering the latter as the result of an evolutionary process and, therefore, as a result of a series of skills acquired by different species at some point in their formation.

Keywords: Animal intelligence. Rationality. animals.

\section{Introducción.}

Investigar si, además de los seres humanos, hay animales que son inteligentes, no resulta una labor sencilla ni trivial. Durante siglos una larga tradición filosófica ha considerado que los seres humanos no son meros animales y se ha establecido una marcada diferencia entre unos y otros. Entre las muchas maneras de distinguir a los seres humanos del resto de los animales se encuentran, por ejemplo, la distinción entre racional e irracional, la atribución de derechos a unos, pero no a los otros, la creencia de que unos son libres mientras que los otros están sujetos a una necesidad natural, la supuesta presencia de un espíritu, alma o principio divino en los seres humanos que está ausente en los animales y muchas otras. El problema con todas estas distinciones es que son artificiales, ya que, si bien podemos identificar algunas diferencias entre los animales y los seres humanos, no debemos olvidar que, a final de cuentas, los seres humanos también son animales. Puesto de este modo, parecería que la pregunta sobre la inteligenia animal es trivial, pues es 
evidente que esas supuestas diferencias podrían ser sólo diferencias de grado, ya que diversos estudios indican que las habilidades que consideramos distintivas de los seres humanos se encuentran también en alguna medida presentes en diversos animales no humanos, entre ellos los elefantes, los primates, los lobos, las hienas, los delfines, los cuervos, los pulpos, los peces y muchos otros. Sin embargo, para una larga tradición filosófica no es para nada clara la inteligencia animal. Lo cierto es que los animales humanos hemos vivido en el planeta Tierra cohabitando con una gran diversidad animales y, si bien algunos de ellos guardan un cierto parecido con nosotros, como los chimpancés, los gorilas, los elefantes, los perros y hasta los delfines; hay otros que parecerían tener muy pocas características similares a las nuestras; tal es el caso de las anémonas, las esponjas marinas, los cuervos, los pulpos, los peces y una gran variedad de insectos, por mencionar sólo algunos. Ante tal diversidad y, dado que los animales humanos son seres inteligentes, es preciso preguntarnos si otros animales son también inteligentes.

\section{Mecanicismo cartesiano y conductismo}

Una de las tradiciones filosóficas más antiguas que niegan que los animales sean inteligentes se remonta a inicio de la modernidad: el mecanicismo cartesiano, que afirma que los animales son meros autómatas que actúan tal como una maquinaria finamente diseñada que es capaz de transmitir información pero que no piensa. Otra es el conductismo de John $B$. Watson, que considera que la vida mental de los animales es una superstición, no accesible al estudio científico, por lo que debemos contentarnos con estudiar el comportamiento observable, que no se considera inteligente sino condicionado. Tal punto de vista ha influido profundamente en el desarrollo de la etología.

La opinión según la cual, para describir científicamente la conducta de los animales, se debe prescindir de cualquier expresión que les atribuya intenciones, pensamientos, deseos y hasta sensaciones, es muy extendida entre los etólogos. Así, no es poco común la creencia de que no hay manera de saber si un animal experimenta algo. Un animal bien podría moverse y actuar del modo en que lo hace en función de la temperatura, la velocidad del viento, la presión atmosférica, la 
intensidad de la luz y otros factores similares sin experimentar absolutamente nada. Así, la mejor opción, se dice, es no atribuir a los animales emociones, ni acciones intencionales, ni sensaciones ni cualquier otra cosa que sugiera que los animales tienen algún tipo de experiencia consciente. Atribuir «intenciones y emociones a los animales, se consideraba una ingenuidad» (De Waal, 2016, p. 13). Así, decir de un perro que se alegra al ver a su amo o que quiere proteger a sus cachorros, se consideraba un sinsentido si lo que se pretendía era describir científicamente la conducta animal. Si algún etólogo mostraba algún interés por la vida interior de los animales, corría el riesgo de ser acusado de acientífico y de antropomorfista.

Sin embargo, esta actitud, aparentemente científica, no lo es en absoluto, ya que tiende a ignorar y hasta a negar la presencia de diversos elementos comunes entre los animales como, por ejemplo, que una gran cantidad de ellos tiene una base cerebral similar a la de los animales humanos, que presentan conductas agresivas en situaciones equiparables o que parecen reconocerse a sí mismos y a otros individuos. Ciertamente debemos evitar interpretar el comportamiento de los animales de un modo antropomórfico, pero no debemos olvidar que a menudo la psicología, la economía, la medicina, la historia y muchas otras ciencias atribuyen a los seres humanos intenciones, creencias, deseos y sensaciones a partir de ciertos datos sobre sus conductas, el volumen de sus intercambios comerciales, sus gestos o los vestigios del pasado que conservan, por mencionar algunos. Surge entonces la pregunta acerca de si está justificado negar definitivamente al resto de los animales aquella actividad mental que sin mucha dificultad atribuimos a los animales humanos, cuando ambos realizan operaciones que son similares. Una propuesta que parece prometedora es partir de la idea de que las habilidades que supuestamente distinguen a los seres humanos están presentes, en alguna medida, en otras especies animales (Safina, 2015, p. 66).

Pese a esto, además de las dos tradiciones que hemos mencionado, hay en filosofía diversos autores que niegan que los animales sean inteligentes y que señalan una diferencia fundamental entre los seres humanos y los demás animales. Si bien no utilizan el término inteligencia, que es 
preferido en las ciencias cognitivas, abordan el problema de la racionalidad y se preguntan si, además de los seres humanos, hay otros animales que sean también racionales.

\section{Davidson}

En el siglo XX, algunos filósofos analíticos han negado la racionalidad animal. Tal es el caso de Donald Davidson, quien, en Animales racionales, luego de afirmar que "ni un bebé de una semana ni un caracol son criaturas racionales" (Davidson, 2003, p. 141), explica que la diferencia entre estos y las personas adultas normales, en cuanto a la racionalidad, "consiste en tener actitudes proposicionales como las creencias, los deseos, las intenciones y la vergüenza" (p. 141). Sin embargo, esto no basta para negar la racionalidad a todos los animales, ya que, si bien los caracoles no parecen tener ninguno de los rasgos que asociamos con la racionalidad, bien podría suceder que otros animales, como, por ejemplo, los delfines, los chimpancés o los perros, fueran racionales. Teniendo esto en cuenta, Davidson se pregunta "qué es lo que hace que un animal (u otra cosa, si uno quiere) sea racional" (p. 142) y enseguida responde que "las actitudes proposicionales proporcionan un criterio interesante de racionalidad" (p. 142) debido, principalmente, a su carácter holista, ya que una creencia, por ejemplo, no puede tenerse de manera aislada, sino que cada creencia requiere de otras actitudes proposicionales, tales como otras creencias, deseos, intenciones y lenguaje. Esto hace que "la distinción entre tenerlas y no tenerlas sea tajante" ( $p$. 142).

Una vez establecido esto, es claro que solamente se puede atribuir justificadamente una creencia si «podemos ubicar los pensamientos dentro de una red de creencias relacionadas, identificamos pensamientos, hacemos distinciones entre ellos y los describimos según lo que son» (p. 145). Esta condición difícilmente podría ser satisfecha cuando inferimos, al observar alguna conducta animal, que esta depende de creencias, intenciones y deseos, ya que, si bien es común explicar la conducta animal apelando a estos elementos, cabría preguntarse si efectivamente los tienen. Decimos, por ejemplo, al ver que un perro persigue un gato, que desea atraparlo; pero, si Davidson está en lo correcto, esto implica que podemos mostrar que el perro tiene, al menos, 
ciertas creencias generales acerca de los gatos y la intención de realizar ciertas acciones para conseguir el fin previsto. Todos estos elementos, a su vez, tendrían que formar parte de una red de pensamientos que presenten un alto grado de coherencia lógica, de modo que "muy pronto llegaremos a creencias sobre las cuales no tendremos idea de si decir que el perro las tiene y que sin embargo son tales que, sin ellas, nuestra confiada atribución primera parece inestable" (p. 145). Esto se debe a que cada actitud proposicional adquiere tanto su contenido como su identidad a partir de muchas otras creencias con las cuales está relacionada. De ahí, Davidson concluye que, aun cuando nada impide que un observador pueda inferir cuáles son las creencias, deseos e intenciones que explican la conducta de un agente, "si una criatura no puede hablar, no está claro que se pueda mantener la intencionalidad en las descripciones de sus supuestas creencias y demás actitudes" (p. 147). Esta conclusión anula la posibilidad de atribuir actitudes proposicionales a cualquier animal que no pertenezca a la especie humana, ya que sin lenguaje resulta imposible atribuir correctamente la presencia de creencias y con ello la de deseos e intenciones a cualquier miembro de otra especie, ya que "solamente los hombres y las mujeres tienen lenguaje, o algo lo bastante parecido a un lenguaje como para justificar la atribución de pensamientos proposicionales" (p. 143)

De acuerdo con lo anterior, para Davidson, para atribuir cualquier pensamiento a un agente "debe haber una buena razón para creer que hay una pauta compleja de comportamiento" (p. 148) y añade que «solamente hay tal pauta si el agente tiene lenguaje» (p. 148). De este modo, no sólo no podemos atribuir ningún pensamiento a un animal que no posea lenguaje, sino que "no puede tener un pensamiento a menos que tenga lenguaje" (p. 148). Y como el lenguaje es una actividad social, podemos agregar que, además de ser capaz de expresar sus pensamientos, cualquier criatura racional debe ser capaz de "interpretar el habla y los pensamientos de los demás" (p. 148), lo que supone, además, la presencia de una gran diversidad de creencias generales verdaderas. Así, aunque usualmente explicamos y predecimos exitosamente "el comportamiento de animales sin lenguaje atribuyéndoles creencias, deseos e intenciones" (p. 149), esto no sería suficiente para justificar tal atribución, debido a que «sería equivocado concluir que estos animales sin habla 
(=incapaces de interpretar o mantener comunicación lingüística) tienen actitudes proposicionales» (p. 149).

Una vez establecido que el lenguaje es una condición necesaria para el pensamiento, Davidson vuelve sobre el argumento de que una criatura no puede ser racional sin actitudes proposicionales y que éstas no pueden darse sin creencias, y agrega que "para tener una creencia es necesario tener el concepto de creencia" (p. 150). Esto significa que, si bien no se requiere que todo pensamiento sea autoconsciente, tener una creencia cualquiera implica, a su vez, que se tiene al menos una creencia sobre alguna creencia, y que sin ella no es posible tener actitudes proposicionales. Si preguntáramos qué tipo de creencias se tienen respecto de otras creencias, Davidson respondería que, para tener un conjunto de creencias que posibilite la presencia de actitudes proposicionales, al menos se requiere que se tengan "creencias sobre la corrección de las propias creencias" (p. 153), y agrega que el concepto de verdad objetiva es necesario para tener el concepto de creencia. Esto se debe a que, para Davidson, el concepto de creencia no puede deslindarse del concepto de verdadero o falso e, incluso, de los conceptos de correcto e incorrecto.

Si esto es así, el que una criatura sea capaz de discriminar entre sensaciones o cambiar su conducta para adaptarla según el aprendizaje previo no "muestra que la criatura domine el contraste entre lo que se cree y lo que es el caso, tal y como se requiere para las creencias" (p. 154) y el lenguaje se presenta como lo único que mostraría el dominio de tal contraste. Esto se debe a que la interacción lingüística posibilita el concepto del mundo intersubjetivo, el cual, finalmente, produce el concepto de verdad objetiva que, como se ha establecido, es necesario para que haya creencias y, en general, pensamientos. Dado esto, Davidson concluye que "la racionalidad es un rasgo social. Solamente la tienen quienes son capaces de comunicarse" (p. 155) y, dado que los animales no son capaces de comunicarse lingüísticamente, no son racionales.

\section{McDowell}

Más recientemente, otro filósofo analítico ha seguido por esta tradición. En la conferencia Los animales racionales y los demás animales, McDowell, luego de denunciar el mito de lo dado y la 
angustia filosófica que provoca la tendencia a explicar la espontaneidad del entendimiento en términos de leyes naturales, se propone buscar "una concepción de nuestra naturaleza que incluya la capacidad de sintonizar con la estructura del espacio de las razones" (McDowell, 2003, p. 177), espacio que es inaccesible a los animales que carecen de capacidades conceptuales. Esto es así, explica, porque el pensamiento conceptual, que depende de la espontaneidad del entendimiento "es lo que saca a escena tanto el mundo como el yo" (p. 182), por lo que las criaturas que carecen de tales capacidades carecen, a su vez, "tanto de autoconsciencia como de experiencia de la realidad objetiva" (pp. 182-183). Y es que, para McDowell, el mundo objetivo solamente puede presentársele a un sujeto que pueda atribuirse a sí mismo experiencias, es decir, a un sujeto autoconsciente cuyas experiencias puedan constituirse como conciencia del mundo (p. 182).

La tesis kantiana defendida por McDowell resulta restrictiva respecto a los meros animales, ya que, al no poseer la espontaneidad del entendimiento, "no podemos considerarlos como si estuvieran continuamente reformando su visión del mundo como respuesta racional a su captación de la realidad" (p. 183). Esta respuesta racional, tal como ha sido caracterizada, "requiere sujetos que se hagan cargo de su pensamiento, que estén listos para evaluar lo que constituye o no una razón para otra cosa y que estén dispuestos a cambiar, de acuerdo con ello, las actitudes de sus respuestas" (p. 183). Tales condiciones, al no ser satisfechas por los meros animales, serían una razón suficiente para negar que estos sean racionales. Además, esas mismas consideraciones son utilizadas por McDowell para poner en duda las capacidades perceptivas de los meros animales y, finalmente, para afirmar que carecen de experiencia externa. Pese a esto, no se suscribe al mecanicismo cartesiano ni niega que los animales tengan percepción y sensación, pero esta obedece exclusivamente a imperativos biológicos sin ninguna relación con la racionalidad (p. 184).

Los meros animales ni siquiera contarían con lo que McDowell llama una subjetividad hecha y derecha, sino tan sólo con una protosubjetividad, ya que si bien "necesitamos, de hecho, recurrir a la sensibilidad del animal ante las características de su entorno si queremos entender su vida atenta y sus movimientos autónomos" (p. 187), luego niega que haga falta atribuirles un comportamiento ante el mundo, y afirma que "en un modo de vida meramente animal, vivir no es sino responder a 
una sucesión de necesidades biológicas" (p. 187). Si esto es así, los meros animales, que carecen de una experiencia externa, también carecerían de una experiencia interna, ya que la sensibilidad no implica que tengan consciencia del mundo externo. De acuerdo con esto, McDowell sostiene que incluso "los sentimientos de dolor o de temor no equivalen necesariamente a una consciencia del mundo interno" (p. 190), por lo que bien podemos negar que un animal posea un mundo interior sin negarle que posea sentimientos.

Aunque McDowell deja claro que no concuerda con la idea cartesiana de que los meros animales sean autómatas, no los cree capaces de tener experiencias, lo que los imposibilita tanto para tomar consciencia del mundo externo como para tener autoconsciencia. Así, la pregunta correcta no es aquella que indaga acerca de si los meros animales son racionales, sino la que plantea "cómo se ha llegado al hecho de que existan animales que posean la espontaneidad del pensamiento" (p. 195).

\section{Etología}

Un enfoque distinto podría equilibrar el modo en que investigamos y describimos la inteligencia humana y animal. En vez de determinar si algún animal es inteligente o no por referencia al animal humano, que es tomado como modelo, Frans de Waal considera que "la comparación no es entre personas y animales, sino entre una especie animal -la nuestra- y una amplia variedad de otras especies" (2016, p. 15). Se trata de eliminar la tajante distinción entre animales y humanos en lo que a la cuestión de la inteligencia se refiere. Si los seres humanos son animales, entonces "no estamos comparando dos categorías separadas de inteligencia, sino considerando la variación dentro de una categoría única" (De Waal, 2016, p. 15). De ser así, la inteligencia humana no es sino una más de las diversas variantes en que se presenta la inteligencia animal. Así lo atestiguan los resultados de diversos estudios provenientes de la etología y de las neurociencias

Desde principios del siglo XX, se han realizado diversos estudios en aves, peces, anfibios, reptiles, primates, perros, ratas, delfines, ardillas y hasta en moluscos, especialmente en pulpos, destinados a investigar la presencia en animales de lenguaje, habilidades numéricas, uso de 
herramientas, reconocimiento de otros individuos, comprensión de relaciones de terceros, autoreconocimiento, comportamiento social, memoria, conciencia y otras habilidades. Los resultados de tales estudios han generado diversas controversias en contra de la creencia generalizada de que los seres humanos son los únicos animales que poseen cultura e inteligencia.

\section{Base cerebral y conciencia}

Un ejemplo paradigmático en torno a la discusión acerca de la posibilidad de conciencia en animales no humanos es la Declaración de Cambridge sobre la conciencia proclamada el 7 de Julio de 2012 en el Churchill College de la University of Cambridge por Philip Low, David Edelman y Christof Koch. Un destacado grupo internacional de neurocientíficos fue reunido "en la Universidad de Cambridge para reevaluar los sustratos neurobiológicos de la experiencia consciente y los comportamientos relacionados en animales humanos y no humanos" (Low, 2012) dando como resultado la siguiente declaración:

La ausencia de neocórtex no parece impedir que un organismo experimente estados afectivos. La evidencia convergente indica que los animales no humanos tienen las bases neuroanatómicas, neuroquímicas y neurofisiológicas de los estados conscientes, así como la capacidad de exhibir conductas intencionales. En consecuencia, el peso de la evidencia indica que los humanos no son los únicos que poseen las bases neurológicas que generan la conciencia. Los animales no humanos, incluidos todos los mamíferos y aves, y muchas otras criaturas, incluidos los pulpos, también poseen estas bases neurológicas (2012). ${ }^{1}$

Si bien la declaración no afirma abiertamente que animales no humanos tengan conciencia, sí sostiene inequívocamente que al menos algunos de ellos tienen una base neurológica similar a la

\footnotetext{
${ }^{1}$ Todas las traducciones son de uno de los autores: Víctor Hugo Gutiérrez. Incluimos el texto original a pie de página con la finalidad de que este pueda ser leído en su lengua original y cotejado. Texto original: The absence of a neocortex does not appear to preclude an organism from experiencing affective states. Convergent evidence indicates that non-human animals have the neuroanatomical, neurochemical, and neurophysiological substrates of conscious states along with the capacity to exhibit intentional behaviors. Consequently, the weight of evidence indicates that humans are not unique in possessing the neurological substrates that generate consciousness. Nonhuman animals, including all mammals and birds, and many other creatures, including octopuses, also possess these neurological substrates.
} 
asociada a la conciencia en animales humanos, así como a la asociada a estados afectivos y que, además, en dichos animales no humanos se observan conductas intencionales. Estos elementos, aun cuando no resuelven el problema acerca de si los animales tienen conciencia, no niegan dicha posibilidad y aportan datos relevantes provenientes de distintas disciplinas científicas que bien podrían ser integrados en una teoría filosófica de la mente animal.

Al respecto, Christof Koch sostiene que muchos animales no humanos poseen un sistema nervioso altamente complejo cuyas "proteínas constitutivas, genes, sinapsis, células y circuitos neuronales son tan sofisticados, variados y especializados como cualesquiera que hayan sido vistos en el cerebro humano" (Koch, 2014, p. 27). ${ }^{2}$ No hay nada excepcional, afirma, ni en la estructura ni en el tamaño del cerebro de los seres humanos. De modo similar, José Luis Díaz sostiene que "dada la conformación anatómica y función cerebral de los diversos animales, y a juzgar por sus habilidades de conducta, se sabe con bastante certeza que tienen percepciones, expresan emociones, resuelven problemas o tienen memoria" (Díaz, 2007, p. 125).

Si bien aún no se han respondido satisfactoriamente muchas de las preguntas acerca de cómo evolucionó el cerebro en los animales, incluidos los humanos; los estudios filogenéticos han dado lugar a diversas teorías que son compatibles con una explicación evolutiva. Resultan particularmente importantes los estudios realizados en primates para comprender las presiones que provocaron el tipo de selección que llevó, por ejemplo, al aumento del tamaño del neocórtex en los primates con respecto al de otros mamíferos. A mediados del siglo XX surge la idea de que lo que favoreció tal incremento de tamaño fue la mera competencia por conseguir una pareja sexual. Sin embargo, la teoría según la cual la compleja vida social es la principal causa del notable crecimiento del neocórtex en los primates ha ido ganando terreno desde que fuera propuesta a mediados de los años sesenta, pero "no es sino hasta 1988, con la postulación de la hipótesis de la inteligencia maquiavélica de Whiten y Byrne, que cobra fuerza la idea de que la vida social de los primates es la causa mayor de la evolución del cerebro" (Mondragón-Ceballos, 2002, p. 29).

\footnotetext{
${ }^{2}$ Texto original: Their constitutive proteins, genes, synapses, cells and neuronal circuits are as sophisticated, variegated and specialized as anything seen in the human brain.
} 
La capacidad de los primates para retener información y manipularla se muestra en la habilidad de estos para identificar objetos y a individuos de la misma especie. Sin embargo, esto es posible gracias a que "la corteza temporal de los primates contiene un mayor número de conexiones con la corteza prefrontal que la que se da en otros mamíferos" (p. 32). A su vez, como ya hemos apuntado, el aumento en el tamaño y la complejidad de las estructuras neurales del cerebro de los primates dependen de la creciente complejidad de su vida social. Hay, pues, una correlación entre el desarrollo del cerebro y la inteligencia maquiavélica de los primates "en el sentido de que los cerebros de gran tamaño (particularmente las neocortezas) son necesarios para el procesamiento de información compleja, pasada y presente, que requiere de una salida conductual igualmente compleja" (p. 33).

Es claro que el cerebro de diversos animales es capaz de procesar información de modos complejos, sin embargo, el verdadero problema radica en saber si las capacidades observadas en animales van acompañadas de una cualidad subjetiva de la experiencia (qualia) como ocurre en los seres humanos. Si bien no tenemos ninguna dificultad para atribuir diversos estados mentales conscientes a otros seres humanos, debemos evitar proyectar en los animales nuestras propias capacidades mentales. Frecuentemente atribuimos tales estados a los animales. Por ejemplo, cuando decimos que el gato siente frío o que el perro quiere salir a correr, atribuimos estados conscientes a estos animales, tal como hacemos cotidianamente con otros seres humanos que, creemos, desean tomar un café o se sienten tristes en un día nublado y frío. Estas y otras actitudes que adoptamos ante la conducta de los animales dependen de nuestro marco emocional y cognitivo hábilmente entrenado para reconocer creencias, deseos, intenciones y sensaciones que, por analogía, son atribuidos tanto a animales humanos como a animales no humanos. Sin embargo, estas actitudes suelen producir inferencias injustificadas cuyo error consiste en atribuir sensaciones, deseos, consciencia, intenciones y otros elementos que comúnmente atribuimos a los seres humanos sin evidencia sólida de que los tienen. En cambio, vale la pena analizar si hay algunos casos en que podamos inferir correctamente que se puedan atribuir tales estados a animales no humanos. 
Experimentos realizados con animales a los que se les administran drogas psicotrópicas han mostrado que algunos mamíferos, entre ellos las ratas, son capaces de distinguir entre dos tipos de drogas distintas. Este resultado, a primera vista trivial, ha sido señalado por José Luis Díaz como un elemento importante en la investigación acerca de la conciencia animal del que se pueden extraer importantes consecuencias.

Los seres humanos son capaces de identificar, correcta y consistentemente, una droga psicotrópica que les ha sido administrada y distinguirla de otras drogas similares porque "reconocen los estados subjetivos que ésta les provoca" (Díaz, 2007, p. 135). Dicha identificación no se lleva a cabo por medio de la observación de su propia conducta y tampoco a través de señalar los receptores bioquímicos que han sido estimulados, sino que se relaciona directamente con la cualidad de la experiencia subjetiva (qualia), "ya que los sujetos reconocen con exactitud cómo se siente o se experimenta el efecto de un fármaco" (p. 136). De este modo, "el reconocimiento sucede porque el sujeto toma conocimiento o reconoce el estado que el fármaco le produce y éste es netamente cualitativo y subjetivo: es un estado de conciencia" (p. 136).

En el caso de los animales que fueron entrenados para activar una palanca cuando una cierta droga les era administrada, cabe preguntarse cómo es que logran identificar que se trata de un mismo fármaco y no de uno distinto o de una solución salina. En una clara analogía al modo en que los seres humanos reconocen la sustancia psicotrópica, José Luis Díaz escribe que:

Debido a que en este tipo de procedimiento no se mide el efecto fisiológico directo del fármaco, sino una conducta que el animal manifiesta cuando está bajo su efecto, esta discriminación podría sugerir y quizás indicar que los animales sienten o perciben el efecto del fármaco y entonces asocian la respuesta con el estado que les produce la droga. Así, el procedimiento no sólo probaría que los animales perciben y asocian un estímulo, sino que lo hacen en forma consciente, es decir, que se percatan de ello. En otras palabras: se podría decir que la conciencia animal ha sido demostrada en términos experimentales (pp. 134135). 
El argumento por analogía es simple, si aceptamos que los seres humanos son capaces de reconocer una droga psicotrópica porque tienen acceso a ciertos estados subjetivos que se presentan cuando dicha droga les es administrada y son capaces de actuar siguiendo tales estados, por qué no aceptar que los animales deben tener también estados subjetivos, de los cuales son conscientes, para poder realizar la acción que ha sido observada en los experimentos. A partir de esta analogía, José Luis Díaz elabora un argumento cuya conclusión está justificada empíricamente y que reproduzco a continuación:

1) La discriminación de drogas en humanos se debe a su efecto subjetivo. 2) La discriminación de fármacos en animales coteja cercanamente con los efectos subjetivos de las drogas en humanos. 3) La interocepción en que se basa la discriminación no se explica sólo por la interacción del fármaco con sus receptores. 4) Conclusión: por analogía, los animales sienten los efectos subjetivos de los fármacos (p. 135).

Por definición, las drogas psicotrópicas son cualquier substancia capaz de influenciar los estados psíquicos, ya que actúan sobre el sistema nervioso, y pueden producir cambios temporales en la percepción, en el ánimo, en los estados de conciencia y en el comportamiento. Es significativo que "sólo aquellas drogas cuyos efectos reconocen conscientemente los humanos son las que pueden discriminar los animales" (p. 136), de modo que cuesta creer que tales animales no sean capaces de inspeccionar y comparar sus propios estados internos de modo similar al de los seres humanos. Así, se puede concluir que "los animales discriminan correctamente los fármacos porque perciben sus efectos psicoactivos específicos" (p. 138) y tal discriminación no puede ser sino consciente. Este argumento implica que ciertos animales tienen experiencias, a diferencia de lo que afirma McDowell. Es por eso que, junto al tema de la conciencia, debemos ocuparnos de otro que resulta de particular importancia en la discusión acerca de la inteligencia animal, tal es el de la sensación. 


\section{Sensación}

Según Koch, inferir que los perros y otros animales experimentan dolor bajo ciertas condiciones está justificado, ya que, en condiciones semejantes, por ejemplo, cuando se está enfermo, estos animales se comportan de manera similar a como se comportan los animales humanos. Y no se trata sólo de una interpretación subjetiva, sino que una medición de ciertos signos fisiológicos parece respaldar dicha inferencia.

Los perros lesionados, al igual que las personas, experimentan un ritmo cardíaco y una presión arterial elevados y liberan hormonas del estrés en su torrente sanguíneo. No digo que el dolor de un perro sea exactamente como el dolor humano, pero los perros, al igual que otros animales, no sólo reaccionan a estímulos nocivos, sino que también experimentan dolor conscientemente (Koch, 2014, p. 26). ${ }^{3}$

Si bien al atribuir estados conscientes como la sensación de dolor a los animales no podemos, sin más, asegurar que los tienen, cabe preguntarse cuál es la actitud más racional cuando se intentan explicar conductas de los animales que parecen suponer una mente consciente. Al respecto, José Luis Díaz, luego de analizar el modo en que algunos etólogos describen la conducta animal, concluye que "la adscripción de estados mentales a los animales no es siempre o necesariamente antropomórfica" (Díaz, 2007, p. 129) y agrega que "la atribución deberá ser tratada como una hipótesis cuya validez puede ser puesta a prueba" (p. 129). Así, al describir la conducta de un perro que coloca la cola entre las patas y huye, se puede suponer que siente miedo, ya que se observa una conexión constante entre dicha conducta y la presencia de una amenaza. Consecuentemente, en una situación de peligro en la que el perro coloque la cola entre las patas se "puede afirmar que el perro tiene miedo, en lugar de llamarlo, por ejemplo, 'conducta ante situación de peligro' o, simple y llanamente, 'cola entre las patas'” (p. 127). Si además de esto consideramos que tenemos ancestros comunes, cerebros similares y conductas análogas a las de

\footnotetext{
${ }^{3}$ Texto original: injured dogs, just like people, experience an elevated heart rate and blood pressure and release stress hormones into their bloodstream. I'm not saying that a dog's pain is exactly like human pain, but dogs-as well as other animals - not only react to noxious stimuli but also consciously experience pain.
} 
muchos animales, cabe preguntarse qué es más racional, si considerar a los animales como completamente diferentes a los seres humanos o considerarlos como parecidos a estos.

Ricardo Mondragón-Ceballos afirma que los primates no solamente son capaces de tener emociones primarias tales como el miedo, el enojo o la tristeza, sino que su estructura cerebral les permite tener emociones secundarias, es decir, emociones esencialmente sociales que “corresponden a la asociación aprendida entre una situación específica con una emoción o conjunto de emociones" (Mondragón-Ceballos, 2002, p. 36) que pueden ser incluso contradictorias entre sí. Estas emociones, si bien son paralelas a cambios corporales autónomos como la secreción de hormonas o cambios en la presión sanguínea, conllevan "una serie de cambios cognoscitivos y perceptuales que conducen a la expresión conductual adecuada" (p. 36).

Además de la discusión que se refiere a la conciencia y a la sensación hay diversos debates en torno a habilidades más específicas, como ejemplo, podemos mencionar la pregunta acerca de si las habilidades numéricas son exclusivas de los seres humanos o se encuentran también presentes en otros animales.

\section{Habilidades numéricas}

Las habilidades numéricas ${ }^{4}$ de algunos animales han sido ampliamente estudiadas desde la década de 1930, principalmente en mamíferos y aves. Sin embargo, en la última década, se ha incrementado significativamente la investigación de habilidades numéricas en peces y "en muchos casos, se han reportado similitudes sorprendentes en el desempeño numérico de mamíferos, aves y peces" (Agrillo \& Bisazza, 2017, p. 1). ${ }^{5}$ Si bien hace falta investigar más acerca del origen genético de

\footnotetext{
${ }^{4}$ El término habilidades numéricas designa la capacidad de discriminar entre cantidades diversas, ya sea en cuanto al número, al orden, a la densidad o al área que ocupan, por mencionar algunas. La presencia de habilidades numéricas no implica necesariamente que se tenga la noción abstracta de número ni la posesión y aplicación de reglas aritméticas sino tan sólo que, de manera intuitiva, se es capaz de discriminar entre dos o más elementos (o conjuntos) de diversas magnitudes.

${ }^{5}$ Texto original: In many cases, surprising similarities have been reported among the numerical performance of mammals, birds and fish.
} 
las habilidades numéricas, con los resultados obtenidos "surge la pregunta acerca de si los sistemas numéricos de los vertebrados se han heredado de un antepasado común" (p. 1). ${ }^{6}$

La capacidad de distinguir y comparar cantidades constituye una ventaja evolutiva, ya que evaluar la cantidad de elementos que tiene un conjunto y compararla entre diversos conjuntos suele ser ventajoso para un animal. Entre las habilidades numéricas que resultan ventajosas para la mayoría de las especies que la poseen, se encuentra "la capacidad de estimar la cantidad de alimentos que están presentes en un sector" (p. 3), ${ }^{7}$ tales como la cantidad de fruta en una rama, el número de cebras en una manada o la cantidad de hormigas rojas que conforman un grupo; tales habilidades facilitan la consecución de alimento a monos, leones y ratones de campo, respectivamente. Otra capacidad que está presente en la mayoría de los animales sociales es la de estimar el número de integrantes que pertenecen a su propio grupo y de compararlo con la cantidad de integrantes de otros grupos de animales de su misma especie. "Los chimpancés, los leones y las hienas, por ejemplo, usan esta habilidad en sus interacciones sociales para decidir si atacan a otro grupo de su misma especie" (p. 3$)^{8}$.

Además, muchas especies adaptan sus tácticas reproductivas a la cantidad de machos y hembras que hay en un grupo. Por ejemplo, los peces mosquito macho prefieren grupos que contienen un mayor número de hembras, lo que probablemente aumenta sus oportunidades de apareamiento (p. 3). ${ }^{9}$ Se sabe también que las hembras de los pavorreales eligen a su pareja, luego de comparar a varios machos, en función de la cantidad de ocelos que tienen en las plumas de su cola. Por otro lado, los peces son más difíciles de identificar por un depredador cuando viven en bancos grandes, además de que, entre más miembros tenga el banco, más fácilmente pueden

\footnotetext{
${ }^{6}$ Texto original: raising the question as to whether vertebrates' numerical systems have been inherited from a common ancestor.

7 Texto original: the ability to estimate the amount of food items that are present in a patch.

${ }^{8}$ Texto original: Chimpanzees, lions and hyenas, for example, use this ability in social interactions to decide whether to attack a group of conspecifics.

${ }^{9}$ Texto original: Male mosquitofish prefer groups that contain a larger number of females, which probably increases their mating opportunities.
} 
detectar depredadores, por lo que "el riesgo de que un pez individual sea capturado disminuye estadísticamente a medida que aumenta la cantidad de individuos en el grupo" (p. 3). ${ }^{10}$

Los estudios con peces indican que estos pueden juzgar la cantidad relativa tan bien como muchas aves y mamíferos. Este resultado sorprende, ya que, debido a que el tamaño del cerebro de los peces es, comparativamente, más pequeño, se esperaría que sus habilidades numéricas fuesen también, más limitadas que las de vertebrados más grandes; sin embargo, esto no es así. Por ejemplo, los peces poecilia reticulata o guppies, son capaces de distinguir el cardumen más grande de entre dos cardúmenes en hasta una razón de 4 a 5 (0.80), y los gasterosteus aculeatus o espinosos, aciertan hasta en una razón de 6 a 7 (0.86); esta proporción es mayor o similar a la reportada en estudios de otras especies tales como perros (0.75), caballos (0.66) y palomas (0.86), pero es inferior a la encontrada en seres humanos $(0.90)$ y monos $(0.87)$ (p. 3). Se ha observado también que los peces distinguen entre cantidades continuas, ya que son capaces de identificar cuál es el cardumen más grande a partir del área, la densidad o la actividad del cardumen. Experimentos en Gambusia affinis o pez mosquito mostraron que puede aprender a discriminar entre cantidades tanto a partir de información numérica, como a partir de información continua, sin que alguna de ellas suponga un mayor esfuerzo cognitivo (p. 4). También han sido estudiadas las habilidades ordinales de los peces y se ha encontrado que, por ejemplo, los guppies "rápidamente aprenden a seleccionar el tercero en una fila de ocho dispensadores de comida diferentes, incluso cuando la distancia y la posición de estos es cambiada sistemáticamente para evitar el uso de señales no ordinales" (p. 4). ${ }^{11}$

Al igual que sucede con los seres humanos, no todos los animales de una especie son igualmente hábiles para resolver problemas numéricos. En un estudio en que se sometió a 8 peces a una serie de tareas cada vez más complejas se encontró que sólo uno pudo distinguir la cantidad más alta en una razón de 2 a 3, dos hasta una razón de 3 a 4 y 5 hasta una razón de 4 a 5 elementos,

\footnotetext{
${ }^{10}$ Texto original: the risk of an individual fish being caught statistically diminishes as the quantity of individuals in the group increases.

${ }^{11}$ Texto original: rapidly learn to select the third position in a row of 8 alternative feeders, even when the distance and position of the feeders are systematically varied to prevent the use of non-ordinal cues.
} 
lo que indica que se pueden encontrar diferencias en las habilidades numéricas entre los individuos de una misma especie (p. 5). Otro estudio revela que la lateralidad del cerebro es un elemento importante para explicar las diferencias en las habilidades numéricas entre individuos de una misma especie. Se dividió a un grupo de peces en tres: lateralizados a la derecha, lateralizados a la izquierda y no lateralizados. Luego se comparó su habilidad para realizar tareas numéricas y no se encontró ninguna diferencia entre los peces lateralizados, ya fuera a la izquierda o a la derecha; sin embargo, los peces no lateralizados se mostraron menos precisos al identificar el cardumen más grande y al distinguir entre diversas cantidades de figuras bidimensionales (p. 5).

Al interpretar los datos obtenidos en los experimentos, se pueden formular dos explicaciones distintas acerca del modo en que los peces distinguen entre dos cantidades distintas. La primera es que se representan de manera abstracta las cantidades involucradas, las cuales son retenidas por un plazo relativamente largo, permitiéndoles elegir la cantidad correcta. La segunda es que los peces identifican el número mayor de entre dos cantidades distintas aprendiendo una regla relacional que aplican a las cantidades involucradas en el experimento. Si bien no se puede descartar la primera explicación, "ahora tenemos evidencia directa de que cuando entrenamos peces para discriminar entre dos cantidades, no codifican información precisa sobre los dos números involucrados; sino que resuelven la tarea aprendiendo una regla relacional” (p. 6), ${ }^{12}$ se trata de la misma estrategia que utilizan los seres humanos en experimentos similares.

Tradicionalmente, se han considerado tres tipos de evidencia experimental para atribuir la capacidad de representación abstracta del número a alguna especie. "El primero es la capacidad de generalizar información numérica a través de variaciones significativas de parámetros físicos no numéricos" (p. 6). ${ }^{13}$ El segundo consiste en la capacidad de identificar una cantidad en una segunda presentación que contiene un conjunto con la misma cantidad además de uno o más distractores

\footnotetext{
${ }^{12}$ Texto original: now have direct evidence that when we train fish to discriminate between two numerosities, they do not encode precise information about the two numbers involved; instead, they solve the task by learning a relational rule.

${ }^{13}$ Texto original: The first is the capacity to generalize numerical information across significant variations in nonnumerical physical parameters.
} 
con cantidades distintas. El tercer tipo de evidencia, "proviene de estudios en los que una especie realiza operaciones aritméticas simples" (p. 6). ${ }^{14}$ Tal evidencia ha sido recopilada por diversos estudios. Por ejemplo, las ratas entrenadas para presionar una palanca frente a dos objetos visuales son capaces de generalizar la regla y aplicarla al mismo número de estímulos auditivos.

Si bien el resultado de tales experimentos no implica que los animales posean la noción abstracta de número, sí supone que son capaces de comparar entre cantidades diversas y de relacionarlas de diversas maneras. Sin embargo, podemos ir más allá y preguntarnos si también hay animales que reconozcan relaciones no numéricas, que podríamos llamar relaciones sociales, ya sea de jerarquía o de parentesco.

\section{Comprensión de relaciones de terceros}

Los elefantes reconocen las relaciones entre terceros. Carl Safina refiere que un elefante hembra Ilamada Tecla, al percibir que el vehículo en que él viajaba se había interpuesto entre una madre y su cría, se da media vuelta, barrita, adopta una actitud amenazadora y llama la atención de la madre, que se acerca corriendo y se coloca frente al vehículo para después reunirse con su cría (Safina, 2015, p. 61). Esta conducta parece implicar que Tecla conoce a la cría y que sabe quién es su madre, es decir, que reconoce la relación que hay entre ellos. Pero los elefantes no son los únicos que reconocen las relaciones que hay entre terceros, sino que también los primates, "así como los lobos, las hienas, los delfines, las aves de la familia de los cuervos y al menos algunos loros" (p. 61). Esto implica que se reconocen a sí mismos, que reconocen a otros miembros de su propia especie y que reconocen las relaciones mantenidas por dichos miembros.

El reconocimiento de relaciones de terceros puede inferirse a partir de algunas conductas complejas que presentan diversos animales, tales como el consuelo, el apoyo o el sabotaje social, tal como se explica a continuación:

En el consuelo, tras un encuentro agresivo, un tercer animal que no participó en la pelea, emite un acto de afiliación a uno de los contendientes. El apoyo se observa cuando

\footnotetext{
${ }^{14}$ Texto original: comes from studies in which a species performs simple arithmetic operations.
} 
dos animales se enzarzan en una pelea y un tercer animal se une a la contienda respaldando a uno de los contendientes en contra del otro. El apoyo se basa a su vez en las alianzas, las cuales se forman a partir de interacciones de afiliación frecuentes. Finalmente, el sabotaje social consiste en deshacer las interacciones de afiliación de terceros por medio de actos que van de agresiones directas a comportamientos aparentemente casuales (MondragónCeballos, 2002, pp. 33-34).

Este tipo de conductas complejas ha sido ampliamente documentado en primates, sin limitarse a ellos, y suponen que son capaces de establecer e identificar relaciones entre terceros. Estas relaciones pueden ser de parentesco, de rango, de jerarquía social, de afiliación, de dominancia o de subordinación, entre otras. Ahora bien, el conocimiento de estas relaciones supone al menos, las siguientes capacidades cognoscitivas: 1) memoria, 2) capacidad de clasificar las diversas relaciones entre individuos, 3) capacidad de inferir relaciones transitivas, 4) reconocer que el intercambio de actos de cierto tipo son socialmente distintos a otros, 5) colegir que tal intercambio de actos puede estar asociado a otros intercambios de actos que incluyan a un tercero y, en tal escenario, 6) decidir disparar o prevenir la ocurrencia de la serie final de actos (Mondragón-Ceballos, 2002, pp. 34-35). La teoría de la inteligencia maquiavélica, al integrar estos resultados, asume que los primates pueden obrar de acuerdo a su propia conveniencia utilizando el conocimiento previo y proyectándolo como una expectativa dirigida hacia el futuro, para ello, pone en práctica diversas estrategias aprendidas en vista a conseguir sus fines. Así, según la evidencia actual, "los primates conocen sus propias relaciones sociales, así como las de terceros y hacen uso de ese conocimiento para orientar su comportamiento social" (p. 34).

Además de los aspectos que hemos mencionado, el relativo a la vocalización de los primates ha sido ampliamente estudiado. Se ha encontrado que estos, además de poseer un amplio repertorio de vocalizaciones, "son capaces de identificar relaciones de parentesco o de dominancia entre terceros a partir de dichas vocalizaciones" (p. 30). Esto nos Ileva naturalmente a la pregunta acerca de si hay animales, además de los seres humanos, capaces de desarrollar, aprender y utilizar 
un lenguaje. Esta pregunta resulta especialmente relevante al considerar el punto de vista de Davidson que hemos analizado anteriormente.

\section{Lenguaje}

Francisco Conesa y Jaime Nubiola señalan que podemos distinguir entre lenguaje humano y comunicación animal atendiendo a cuatro elementos. En primer lugar, la comunicación animal es instintiva e involuntaria, mientras que "en el habla humana no hay una base instintiva apreciable" (Conesa \& Nubiola, 2002, p. 21). En segundo lugar, mientras los animales utilizan un sistema de comunicación icónico, en el que la relación entre el mensaje y la señal es relativamente simple y directa; el lenguaje humano es en su mayoría dígito, ya que la relación entre los signos utilizados y el mensaje es arbitraria, utilizando un número limitado de signos se pueden construir un número infinito de palabras (pp. 21-23). En tercer lugar, la comunicación animal cuenta con una productividad limitada y se presenta ante un contacto directo con las condiciones o acontecimientos a que se refiere; en cambio, el lenguaje humano es infinitamente productivo, su universalidad semántica le permite transmitir información sobre aspectos presentes o ausentes, pasados o futuros, y hasta se puede nombrar lo desconocido, todo esto sin necesidad de contar con la presencia de aquello a lo que se refiere una expresión lingüística (p. 23). Por último, en la comunicación animal no se ha encontrado que los símbolos utilizados puedan referirse a ellos mismos, mientras que el lenguaje humano puede referirse a sí mismo, es decir, posee reflexividad o capacidad metalingüística. Las diferencias señaladas llevarían, según estos autores, a concluir que “el lenguaje compete de manera propia e intrínseca únicamente al ser humano" (p. 24).

Pese a lo anterior, la pregunta acerca de si los meros animales poseen lenguaje ha generado un debate que se ha visto avivado por los diversos intentos de enseñar lenguaje a algunos primates desde los años sesenta. En los primeros experimentos, se intentó enseñar el lenguaje de los sordomudos a algunos chimpancés. Washoe, un chimpancé hembra, logró utilizar hasta 132 signos, mientras que un gorila hembra llamada Koko llegó a usar 300 signos. En proyectos posteriores se remplazó el lenguaje de los sordomudos por lexigramas, es decir, símbolos geométricos de plástico 
que se hacían corresponder con palabras de un vocabulario humano, Lana, otr chimpancé hembra, llegó a usar hasta 200 de estos símbolos, mientras que Sherman y Austin lograron comunicarse entre ellos a través de los lexigramas. Kanzi, un bonobo, además de utilizar los lexigramas con pericia, aprendió a reconocer algunas frases del inglés. Estos y otros estudios llevaron a pensar que algunos primates tienen la capacidad de comunicarse a través de símbolos y de utilizar una sintaxis, lo cual apunta a que son capaces de utilizar un lenguaje que se parece bastante al lenguaje humano. Sin embargo, el debate continúa entre los que defienden la capacidad de simbolización de los primates y aquellos que señalan la discontinuidad radical entre el modo en que los primates utilizan los símbolos y el lenguaje humano (p. 20).

\section{Referencias}

Agrillo, C., \& Bisazza, A. (2017). Understanding the origin of number sense: a review of fish studies. Philosophical Transactions B, 373(20160511), 1-13.

Conesa, F., \& Nubiola, J. (2002). Filosofía del lenguaje (Segunda ed.). Barcelona, España: Herder. Davidson, D. (2003). Subjetivo, intersubjetivo, objetivo. (O. Fernández Prat, Trad.) Madrid, España: Cátedra.

De Waal, F. (2016). ¿'Tenemos suficiente inteligencia para entender la inteligencia de los animales? (A. García Leal, Trad.) Barcelona, España: Tusquets.

Díaz, J. L. (2007). La conciencia viviente. México D.F., México: Fondo de Cultura Económica. Koch, C. (Enero-Febrero de 2014). Ubiquitous Minds. Scientific American Mind, XXV(1), 26-29.

Low, P. (2012). The Cambridge Declaration on Consciousness. Declaración, The University of Cambridge, Cambridge.

McDowell, J. (2003). Mente y mundo. (M. Á. Quintana Paz, Trad.) Salamanca, España: Sígueme. Mondragón-Ceballos, R. (Octubre de 2002). La inteligencia maquiavélica de los primates y la evolución del cerebro social. Salud mental, 25(5), 29-39.

Safina, C. (2015). Mentes maravillosas. Lo que piensan y sienten los animales. (I. Oliva Luque, I. Clavero Hernández, \& P. Aguiriano Aizpurua, Trads.) Barcelona, España: Galaxia Gutenberg. 